\title{
Achieving High Cancer Control Trial Enrollment in the Community Setting: An Analysis of the Community Clinical Oncology Program
}

\author{
Sara R. Jacobs ${ }^{1, \S}$, Bryan J. Weiner ${ }^{2}$, Lori M. Minasian ${ }^{3}$, and Marjorie J. Good ${ }^{3}$ \\ ${ }^{1}$ Department of Health Policy and Management, Gillings School of Global Public Health, \\ University of North Carolina at Chapel Hill, North Carolina, United States \\ ${ }^{2}$ Cecil G. Sheps Center for Health Services Research, University of North Carolina at Chapel Hill, \\ North Carolina, United States \\ ${ }^{3}$ Division of Cancer Prevention, National Cancer Institute, Bethesda, MD, United States
}

\section{Abstract}

Determining the factors that lead to successful enrollment of patients in cancer control clinical trials is essential as cancer patients are often burdened with side effects such as pain, nausea, and fatigue. One promising intervention for increasing enrollment in cancer control trials is the National Cancer Institute's Community Clinical Oncology Program (CCOP). In this article, we examined CCOP staffing, polices, and procedures associated with enrollment in control trials. Data were obtained from three sources: the online CCOP, MB-CCOP, and Research Base Management System, CCOP Annual Progress Reports, and a survey of CCOP Administrators conducted in 2011. We analyzed cancer control trial accrual in 2011 among 46 CCOPs using multivariate regression. Three factors were significant predictors of accrual. First, having a team of staff dedicated to enrolling patients in control and prevention trials, compared to having no dedicated staff, was associated on average with an additional 30 patients enrolled in control trials ( $\mathrm{p}<0.05$ ). Second, CCOPs that recognized physicians for enrolling a large number of patients compared to CCOPs that did not recognize high enrolling physicians enrolled on average an additional 25 patients in control trials $(\mathrm{p}<0.05)$. Lastly, the number of cancer control trials available was also associated with enrollment $(\beta=5.50, p<0.00)$. Our results indicate that CCOPs looking to increase enrollment in control trials should consider dedicating a team of staff to enroll patients in these types of trials. In addition, CCOPs or other volunteer research systems looking to increase physician participation should consider recognizing high enrolling physicians.

\section{Keywords}

Cancer; Clinical trial enrollment; Cancer symptom control; Community Clinical Oncology Program

(C) 2012 Elsevier Inc. All rights reserved.

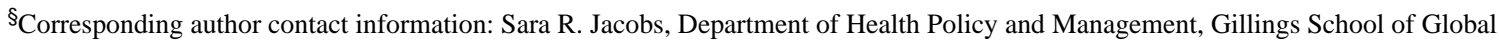
Public Health, 1101 McGavran-Greenberg Hall, CB \#7411, University of North Carolina at Chapel Hill, Chapel Hill, NC 27599-7411, Phone: 513-543-2637, Fax: 919-966-6961, jacobssr@live.unc.edu.

Publisher's Disclaimer: This is a PDF file of an unedited manuscript that has been accepted for publication. As a service to our customers we are providing this early version of the manuscript. The manuscript will undergo copyediting, typesetting, and review of the resulting proof before it is published in its final citable form. Please note that during the production process errors may be discovered which could affect the content, and all legal disclaimers that apply to the journal pertain. 


\section{Introduction}

Despite evidence that clinical trials play a critical role in developing innovative treatments and in refining cancer prevention and control strategies, only 3-5\% of adults with cancer in the United States participate in clinical trials [1]. One promising intervention for increasing participation in cancer clinical trials is the Community Clinical Oncology Program (CCOP). The CCOP network is a joint venture between the National Cancer Institute (NCI)'s Division of Cancer Prevention, which provides overall direction and funding, research bases, which design clinical trials, and community-based networks of providers (CCOPs), which assist with enrollment, data collection, and dissemination of study findings [2-8]. Although all three components of the network are critical to the success of the national program, the goal of this analysis is to identify the CCOP staffing, polices, and procedures associated with enrollment in NCI-sponsored cancer control trials. As defined by CCOP, cancer control trials test the effectiveness of symptom management, rehabilitation, and continuing care interventions to minimize cancer burden and improve quality of life [9].

Determining the factors that lead to successful enrollment of patients in cancer control trials is important for clinical care [10]. Among patients with advanced cancer, the majority experience moderate to severe pain, fatigue, anorexia, and/or nausea $[11,12]$. These side effects may persist even after active treatment ends. Efforts to improve side effects have not kept pace with efforts to develop innovative cancer treatments [13]. As the number of cancer survivors continues to grow, addressing the burden of cancer related side effects will continue to be important [13].

Understanding the factors that lead to successful enrollment of patients in control trials is also important for CCOP administrators. The landscape of available clinical trials is changing. In the past, CCOPs met a significant portion of NCI accrual expectations through large-scale cancer prevention trials [2]. For example, CCOPs overall provided approximately $30 \%$ of the enrollment in the Breast Cancer Prevention and the Selenium and Vitamin E Cancer Prevention Trials [2]. As the number and scale of prevention trials decrease, there is an increasing need to meet accrual expectations through enrollment in cancer control clinical trials.

Prior research of cancer control trial accrual in the CCOP network has examined control and prevention trial enrollment together $[3,4,5,6]$. Quantitative analyses have focused on fixed organizational structural and environmental factors that contribute to enrollment [3,4]. CCOP administrators, however, are unable to modify these factors to increase enrollment in clinical trials. There have also been a number of case studies of successful CCOPs [5,6]. These studies tend to focus on prevention enrollment. Although some of the strategies also apply to control trials (e.g., dedicated research staff), others are not as relevant (e.g., mass media campaigns) [5].

In this article, we examine modifiable CCOP staffing, operational polices, and procedures to determine their effect on enrollment in cancer control trials. We believe our results are relevant beyond CCOPs to other clinical research programs conducted within community settings. Our findings are particularly applicable for volunteer research programs hoping to encourage physician participation in clinical research.

\section{Materials and Methods}

\section{Study Setting and Population}

The study population is the NCI CCOP network. As of November 2012, 47 CCOPs and 17 Minority-based CCOPs (MB-CCOPs) operated in 35 states and Puerto Rico. MB-CCOPs 
primarily focus on enrolling minority patients into NCI-sponsored clinical trials. MBCCOPs also tend to be located primarily at academic medical centers or universities, whereas university hospitals may be part of some CCOPs, they cannot be the lead organization. Therefore, MB-CCOPs also tend to be located in more urban areas than participating CCOPs, which focus on enrolling patients in a local or community setting. We had to exclude MB-CCOPs from this analysis, as T-tests and Chi $\mathrm{X}^{2}$ tests demonstrated that they are systematically different than the CCOPs. For example, CCOPs on average enrolled over 108 patients to cancer control trials while MB-CCOPs only enrolled 37 patients. In addition, the number of available cancer control trials was on average 21 trials for CCOPs, but only 10 trials on average for MB-CCOPs. Both differences were statically significant. Thus, we did not feel comfortable combining both CCOPs and MB-CCOPs in a single analysis.

The specific sample for this study includes 46 CCOPs, as an additional CCOP joined the program after the data was collected. In total, the CCOP network includes over 450 hospitals and physician practices, with the average CCOP comprised of about 10 hospitals or practice sites. CCOPs also include over 2,000 physicians, with the average CCOP composed of 48 physicians.

\section{Study Design and Data Sources}

The study used a cross-sectional design with the CCOP as the unit of analysis. We obtained data from three sources. The online CCOP, MB-CCOP, and Research Base Management System, maintained by NCI Division of Cancer Prevention, provided data on CCOPs' 2011 menu of NCI-sponsored cancer control trials and CCOPs' 2011 patient enrollment into those trials. Second, the progress reports that CCOPs submit annually to NCI provided data on the CCOP's cancer patient volume. The progress reports covered the nine-month period from June 2010 through February 2011. Finally, a survey of CCOP Administrators conducted in the fall of 2011, provided data on the total number of CCOP staff (including CCOP-funded and non-CCOP funded staff) in 2011, cancer control and prevention dedicated staff in 2011, and whether the CCOP recognizes physicians for enrolling patients and/or expects physicians to enroll a certain number of patients per year. The goal of the survey was to learn more about how the CCOPs are organized and how they operate. The survey specifically addressed CCOP organizational structure, sponsored educational trainings, physician resources and support for screening, consenting, and enrolling patients, as well as CCOP staffing procedures. The survey was designed and administrated with the support of NCI Division of Cancer Prevention officials. Although the time periods covered by the three data sources do not overlap perfectly, the CCOP features examined in this study exhibit only small fluctuations from year to year.

\section{Measures}

The study's outcome was patient enrollment (i.e., accrual) in NCI-sponsored cancer control trials in 2011. We did not include accrual into cancer prevention trials. Given the goal of this article was to determine CCOP staffing, organizational polices, and procedures that influence cancer control trial enrollment, we selected two sets of factors that we felt CCOP administrators could modify and would influence future planning and CCOP operations.

The first factor characterizes the CCOP's staffing arrangement and included two measures: (1) Number of CCOP research staff in 2011, defined as the number of non-physician personnel supported by CCOP research grants and the number of staff who worked on NCI CCOP trials who were not supported by CCOP funds (e.g., whose salaries were covered by participating hospitals); and (2) Number of CCOP research staff dedicated to control and prevention trials who focused on enrollment in NCI-sponsored cancer control and 
prevention trials in 2011. We were unable to distinguish cancer control dedicated staff from those focused on prevention. Given the average enrollment in cancer prevention trials was only nine patients and CCOPs enrolled on average 109 patients in cancer control trials, however, it is likely that these staff focused a significant portion of their time on cancer control enrollment. This measure was assessed as having no dedicated staff, one staff member, or more than one staff member dedicated to cancer control and prevention trial accrual. We decided to look at cancer control and dedicated staff this way, as we wanted to compare having a small or large staff to having no dedicated staff. Including dedicated staff as a continuous variable would only allow us to examine the effect of adding an additional staff person on total enrollment.

The second factor characterizes the CCOP's organizational policies and procedures. The first two measures included: (1) Recognition of physicians for enrolling patients, and (2) Expectation for physicians to enroll patients. Recognition of high enrolling physicians was defined as whether the CCOP provided some form of recognition to physicians with high levels of accrual to NCI-sponsored clinical trials (e.g., public acknowledgement, small tokens of appreciation) in 2011, assessed as yes or no. Expectations for enrollment was defined as whether the CCOP expected physicians to enroll a minimum number of patients in any type of NCI-sponsored clinical trial, including formal and informal communications in 2011. This measure was also assessed as yes or no. We included these CCOP policies, which focus on the accruing physicians, in the analysis because although CCOP support staff are instrumental in helping to recruit and consent patients, ultimately accrual to all types of clinical trials is attributed to individual physicians. Thus, CCOP-affiliated physicians may be more involved in cancer control trial enrollment than are physicians in other settings, making these policies potentially relevant factors that influence patient enrollment in cancer control trials. In addition, some physicians may be less likely to enroll patients in cancer treatment trials compared to cancer control trials, as they may be more likely to disagree with the specific protocol. For example, in an interview with a CCOPaffiliated physician, the physician explained he was much more comfortable putting patients on cancer control trials and tended to refer patients to these types of trials compared to treatment trials. Therefore, implementing expectations for enrollment or recognizing high enrolling physicians are modifiable CCOP organizational policies that may drive cancer control trial enrollment.

The last CCOP organizational procedure is: (3) Number of open NCI Cancer Control trials for which the CCOP had at least one patient enrolled in 2011. Given our measure for the number of open cancer control trials included only trials with at least one patient enrolled, we ran a series of sensitivity test to ensure it was robust to small variations in the total number of open trials. The results from these tests are described below. Lastly, we also tried to control for differences in CCOP size by including one additional measure: (1) Number of newly diagnosed cancer patients seen in participating CCOP hospitals in 2009 (the most recent year of data available for this measure for this study).

\section{Statistical Analysis}

We utilized ordinary least squares (OLS) multivariate regression models to analyze our results. OLS allows us to explain the total enrollment in cancer control trials according to the variables selected above by fitting a line through data in such a way that the sum of the vertical lines from the data to the fitted line, squared, are minimized [14]. To use OLS appropriately, several assumptions must be met. Two assumptions that warrant testing are the assumption of homoskedasticity (i.e., constant variance for all observations) and the assumption that the error term is normally distributed. Although neither of these assumptions bias our parameter estimates, further adjustments to the model may be necessary to ensure our significance tests are valid [15]. We tested for non-normality of the 
error term by running a skewness and kurtosis test for normal distribution, and for homoskedasticity by running the White test for homoskedasticity. Our data was both nonnormal and heteroskedastic. We therefore, bootstrapped our data at 1,000 repetitions and used the $95 \%$ bias-corrected confidence intervals to determine the significance and the marginal effects for each included variable. All analyses were conducted in Stata 12 (StataCorp. 2009. Stata Statistical Software: Release 12. College Station, TX: StataCorp LP).

\section{Results}

In 2011, CCOPs accrued an average of 109 patients in NCI-sponsored cancer control trials (Table 1). Cancer control accrual ranged from 17 to 407 patients. On average, CCOPs had approximately 21 open cancer control trials with at least one patient enrolled. The mean number of newly diagnosed cancer patients per year was 5802. The mean number of total research staff per CCOP was 19. The mean number of CCOP research staff dedicated to enrolling patients in cancer control and prevention trials was 3.6. About $61 \%$ of CCOPs recognized physicians for enrolling patients into clinical trials and about $35 \%$ had informal or formal expectations communicated to physicians on the number of patients they should enroll in trials each year.

Bootstrapped OLS results indicate that there were three significant predictors of cancer control trial accrual (Table 2): (1) number of open cancer control trials ( $p<0.00),(2)$ having more than one research staff member dedicated to enrolling patients in cancer control and prevention trials $(\mathrm{p}<0.05)$, and (3) CCOP recognition of physicians for enrolling patients in clinical trials $(\mathrm{p}<0.05)$. The bias-corrected marginal effects indicate that a one-trial increase in the number of open cancer control trials can lead to on average an additional 5.5 patients enrolled in cancer control trials. Having more than one staff member dedicated to cancer control and prevention trial enrollment compared to having zero staff members dedicated to cancer control and prevention trials is associated with on average an additional 30 patients enrolled. Lastly, CCOPs that recognized physicians who enrolled a large number of patients into clinical trials, compared to those that did not, can lead on average to an additional 25 patients enrolled in cancer control trials.

\section{Sensitivity Tests}

Due to data constraints, our measure of the number of open cancer control trials included only those trials for which a CCOP had at least one patient enrolled. We ran a series of sensitivity tests to assess the robustness of our results to different assumptions regarding the number of additional cancer control trials a CCOP had open that had no enrollment. In the first three scenarios, we assumed that all CCOPs had the same percentage of cancer control trials with no accrual. In these models, we increased the number of open cancer control trials for all CCOPs by $10 \%, 20 \%, 30 \%$ respectively. In the fourth scenario, we assumed that CCOPs varied randomly in the percentage of open cancer control trials with no accrual. In this model we randomly increased the number of open cancer control trials across CCOPs from $0 \%$ to $20 \%$. In the final scenario, we assumed that higher performing CCOPs were more "efficient" than lower performing CCOPs in terms of only opening trials that had a strong probability of accrual or in terms of quickly closing trials that exhibited no accrual. For this model, we increased the number of open cancer control trials by $0 \%$ for CCOPs in the highest quintile of cancer control accrual, $5 \%$ for CCOPs in the second highest quintile, $10 \%$ in the third highest quintile, and so on. In all five sensitivity tests, we obtained nearly identical results to those reported above. 


\section{Discussion}

Our goal was to identify the CCOP staffing, organizational polices, and procedures associated with patient enrollment in cancer control clinical trials. We found three significant factors. First, having dedicated cancer control and prevention staff was a significant determinant of enrollment. Enrolling patients in cancer control trials was not dependent on the size of the total CCOP staff, but rather was dependent on the size of the dedicated staff of the CCOP. For example, the total number of CCOP staff was not a significant predictor of cancer control trial enrollment. In addition, CCOPs with only one staff member dedicated to cancer control and prevention trials did not significantly enroll more patients in cancer control trials than CCOPs who had no dedicated staff members. CCOPs with a team of staff dedicated to cancer control and prevention, however, enrolled on average an additional 30 patients compared to CCOPs with no dedicated staff.

Enrolling patients in cancer control trials requires a team of staff dedicated to identifying, recruiting, enrolling, and administrating the trials. Our results support the notion that the degree of work required to accrue to cancer control trials is larger than one person can manage. For example, cancer control studies tend to have more patient reported forms to complete, which can take more time and coordination among staff. Cancer control trials are also generally shorter in duration than treatment trials, but the work involved while a patient is on a study may be more time consuming, which would take a single cancer control staff person away from recruitment efforts. In addition, having only one or no dedicated cancer control staff is likely reflective of the importance a CCOP places on accruing to cancer control trials. Although our analysis was not able to take into account the varying knowledge and/or skill of the cancer control dedicated staff, our results are indicative that successful cancer control accrual is dependent on having a team of staff in place.

Although physicians may play less of a predominant role in cancer control trial accrual than they do in treatment trial accrual, given their primary focus of treating cancer, recognizing physicians for enrolling patients can make a significant impact on CCOP cancer control trial accrual. CCOPs that recognized high enrolling physicians compared to those that did not recognize physicians, on average accrued an additional 25 patients to cancer control trials. One reason that providing recognition for top physician accruers may be a significant predictor of cancer control enrollment is that although physicians are never paid directly for their recruitment efforts, physicians are competitive by nature and recognition in front of their peers is an effective motivator to approach and enroll eligible patients. For example, in interviews with one CCOP in the Midwestern United States, the research staff explained that they hold an annual competition among physicians to see who can enroll the most patients. The CCOP awards an actual crown at the annual Christmas party to the physician with the highest number of patients enrolled in clinical trials. Clinical research staff at this CCOP noted that this competition is an effective motivator for their physicians. Although most physicians will not win or come close to winning, the competition is a motivator for recruitment, as they do not want to come in last place. In addition to motivating physicians to enroll patients, the competition also keeps enrollment and clinical trials top of mind.

Physicians may receive both informal and formal recognition for their accrual efforts. For example, when a physician enrolls a patient in a cancer control trial, they may be immediately recognized and appreciated informally by cancer control support staff. These interactions between dedicated cancer control staff and physicians strengthen the overall recruitment efforts of the team. The majority of CCOPs also provide some form of formal recognition for physicians that enroll a large number of patients. There are two main ways CCOPs recognize physicians for their accrual efforts. The first is providing some sort of public recognition. Most of the CCOPs that recognized doctors publically acknowledged top 
accruers for example, in monthly newsletters, at research or annual meetings, or on public websites. Some of the CCOPs that recognized accrual efforts provided small tokens or gifts to top enrollers such as a coffee gift card, gift basket, or a plaque/trophy. Many CCOPs that recognized physicians did a combination of both public acknowledgement and a small gift or trophy. Therefore, CCOPs or any other clinical trial programs in the community setting that want to increase the enrollment of patients may want to consider adding some sort of small reward, competition, or public acknowledgment of top enrolling physicians. Other volunteer research systems may also want to consider recognizing physicians as a way to increase physician involvement and motivation, as it can be a simple, effective, and easy system to implement.

Lastly, the number of open cancer control trials with at least one patient enrolled was also a significant predictor of enrollment of CCOPs. It is not surprising that having access to a large menu of available cancer control trials can lead to more patients enrolled. Although the number of open trials is significant, an increase of one available cancer control trial was only associated on average with an additional 5.5 patients enrolled overall. Five additional patients is far less than the other two significant factors, recognition and cancer control dedicated staff, where each contributed to over 25 additional patients enrolled.

A number of factors included in the analysis were not significant predictors of cancer control accrual. First, the volume of newly diagnosed patients at the CCOP was not a significant predictor of cancer control enrollment. Originally, we thought having more newly diagnosed patients would give CCOPs more chances to enroll patients in cancer control trials. This was not the case, however. This factor may not have been significant because it is not a true reflection of the number of newly diagnosed patients that the CCOP participating physicians actually treat and are eligible for a clinical trial. Our estimate of the number of newly diagnosed patients comes from the CCOP Annual Progress Reports, which generally use information from the hospital-based registry and thus do not take into account the actual number of patients referred or accessible to a CCOP participating physician. Thus it may be insignificant due to a measurement problem. In addition, the number of newly diagnosed patients may be more applicable in other studies that examine the predictors of treatment trial accrual compared to our analysis where we focused exclusively on cancer control accrual.

Having an informal or formal expectation for physicians to enroll a certain number of patients was also not a significant predictor of cancer control accrual. This may be because the majority of CCOPs do not set any expectations for enrollment. In addition, among CCOPs that did have some sort of formal or informal expectation, the requirement was low, generally just several patients per year. Given that the requirements are low and not specific to cancer control trials, the majority of physicians may be fulfilling any requirements by enrolling patients in treatment trials.

\section{Limitations}

As with any analysis, there are a number of limitations. First, we only have a sample size of 46 CCOPs. Although OLS regression can work with small samples, ideally the sample size should be at least about 10 times the number of explanatory variables, which in our case is at least a sample size of 60 CCOPs [14]. Although 60 CCOPs would be ideal, there are a limited number in the program. In addition, if we had excluded potentially relevant variables in the analysis due to sample size, our estimates would be biased. For example, total combined staff is significantly positively correlated both with the outcome, cancer control enrollment, and the independent variable, number of available cancer control trials. If we had left out total combined staff our coefficients estimates would be biased upwards and 
thus not reflect an accurate portrayal of the factors that influence cancer control enrollment [15].

In addition, this analysis is cross-sectional and only represents a snapshot of the cancer control enrollment and the number of available cancer control trials at a given point in time. This study is unique and significant, however, as much of the data comes from the CCOP Administrator Survey, which contains information never before collected and analyzed about the CCOP program.

Overall, this analysis highlights the importance of conducting clinical trials in the community setting. Although cancer treatment trials are imperative for the development of innovative cancer therapies, this paper emphasizes trials to reduce suffering and increase the quality of life for current cancer patients. Our results are meaningful in that they provide tangible strategies that are implementable for practice administrators to increase physician participation in volunteer research programs and extend the oncologist's role beyond the role of only a treatment provider, and into a role that includes caring for their cancer patient across the entire care continuum.

\section{Conclusion}

Although cancer clinical trials are important in the development of new treatments and innovative cancer control and prevention strategies, only 3-5\% of American adults with cancer participate [1]. One strategy aimed at increasing enrollment, the community-based CCOP network, has been successful in increasing enrollment specifically in cancer symptom management trials [2]. In this analysis we examined the impact of modifiable CCOP staffing, organizational polices, and procedures on the enrollment in NCI-sponsored cancer control related trials. The two largest determinants are (1) having a staff dedicated to cancer control and prevention trial enrollment and (2) recognizing high enrolling physicians for their accrual efforts, both of which on average increased enrollment in cancer control trials by over 25 patients each. CCOPs wanting to increase enrollment specifically in cancer control trials may want to consider dedicating a team of staff to enroll patients in cancer control trials as well as recognizing high enrolling physicians. These results are applicable beyond CCOPs and may be useful for other volunteer research systems looking to increase physician motivation and involvement.

\section{Acknowledgments}

This work was supported by the National Cancer Institute at the National Institutes of Health (R25CA116339 and R01CA124402).

\section{Abbreviations}

$\begin{array}{ll}\text { NCI } & \text { National Cancer Institute } \\ \text { CCOP } & \text { Community Clinical Oncology Program } \\ \text { MB-CCOP } & \text { Minority-based Community Clinical Oncology Program }\end{array}$

\section{References}

1. National Cancer Institute. [Accessed 18 July 2012] Cancer Clinical Trials: The Basic Workbook. Available from: http://www.cancer.gov/clinicaltrials/resources/basicworkbook/

2. Minasian LM, Carpenter WR, Weiner BJ, et al. Translating research into evidence-based practice: the National Cancer Institute Community Clinical Oncology Program. Cancer. 2010; 116:4440 4449. [PubMed: 20572032] 
3. Kaluzny AD, Lacey LM, Warnecke R, et al. Accrual of patients to randomized clinical trials. Factors affecting cancer prevention and control research. International Journal of Technology Assessment in Health Care. 1994; 10:506-516. [PubMed: 8071011]

4. Carpenter WR, Fortune-Greeley AK, Zullig LL, et al. Sustainability and performance of the National Cancer Institute's Community Clinical Oncology Program. Contemporary Clinical Trials. 2012; 33:46-54. [PubMed: 21986391]

5. McKinney MM, Weiner BJ, Carpenter WR. Building community capacity to participate in cancer prevention research. Cancer Control. 2006; 13:295-302. [PubMed: 17075567]

6. McKinney MM, Weiner BJ, Wang V. Recruiting participants to cancer prevention clinical trials: lessons from successful community oncology networks. Oncology Nurse Forum. 2006; 33:951-959.

7. Teal R, Bergmire DM, Johnson M, et al. Implementing community-based provider participation in research: an empirical study. Implementation Science. 2012; 7(1):41. [PubMed: 22568935]

8. Carpenter WR, Weiner BJ, Kaluzny AD, et al. The effects of managed care and competition on community-based clinical research. Medical Care. 2006; 44:671-679. [PubMed: 16799362]

9. National Cancer Institute. [Accessed 24 July 2012] Cancer Clinical Trials: Clinical Trial Definitions. Available from: http://www.cancer.gov/cancertopics/factsheet/Information/clinicaltrials

10. Shoemaker LK, Estfan B, Induru R, et al. Symptom management: An important part of cancer care. Cleveland Clinical Journal of Medicine. 2011; 78(1):25-34.

11. Donnelly S, Walsh D. The symptoms of advanced cancer. Seminars in Oncology. 1995; 22(2 suppl 3):67-72. [PubMed: 7537907]

12. Walsh D, Rybicki L, Nelson KA, et al. Symptoms and Prognosis in advanced Cancer. Support Care Cancer. 2002; 10:385-388. [PubMed: 12136221]

13. National Institutes of Health. [Accessed 13 November 2012] State of the Science Conference Statement. Symptom Management in Cancer: Pain, Depression and Fatigue. Available from: http://consensus.nih.gov/2002/2002CancerpainDepressionFatiguesos022html.htm

14. Wooldridge, JM. Introductory Econometrics: A Modern Approach. 4. Ohio: SouthWestern; 2009. 15. Agresti, A.; Finlay, B. Statistical Methods for the Social Sciences. 3. Prentice Hall; 1997. 
Table 1

Summary Statistics of CCOP Enrollment in NCI-Sponsored Cancer Control Trials, 2011

\begin{tabular}{|c|c|c|}
\hline & $\begin{array}{l}\text { Sample Mean or Percent* } \\
\text { Entire Sample }(n=46)\end{array}$ & Range (Min, Max) \\
\hline \multicolumn{3}{|l|}{ Dependent Variable } \\
\hline Patient Enrollment in NCI- Sponsored Cancer Control Trials & $108.67(91.36)$ & $(17,407)$ \\
\hline \multicolumn{3}{|l|}{ Independent Variables } \\
\hline Number of Open Cancer Control Trials & $20.83(9.92)$ & $(5,45)$ \\
\hline Number of Newly Diagnosed Patients & $5801.80(4923.84)$ & $(238,28746)$ \\
\hline Total Number of CCOP Staff & $19.14(13.36)$ & $(2,79.7)$ \\
\hline $\begin{array}{l}\text { Total Number of Staff Dedicated to Enrolling Patients in Cancer Prevention and } \\
\text { Control Trials }\end{array}$ & $3.63(5.66)$ & $(0,26)$ \\
\hline CCOPs with No Staff Dedicated to Prevention and Control Trials & $23.91 \%$ & \\
\hline CCOPs with One Staff Member Dedicated to Prevention and Control Trials & $19.57 \%$ & \\
\hline $\begin{array}{l}\text { CCOPs with More than One Staff Member Dedicated to Prevention and Control } \\
\text { Trials }\end{array}$ & $56.52 \%$ & \\
\hline \multicolumn{3}{|l|}{ Recognition of Physicians for Enrolling Patients into Clinical Trials } \\
\hline Yes & $60.87 \%$ & \\
\hline No & $39.13 \%$ & \\
\hline \multicolumn{3}{|l|}{ Expectations for Physicians to Enroll Patients into Clinical Trials } \\
\hline Yes & $34.78 \%$ & \\
\hline No & $65.22 \%$ & \\
\hline
\end{tabular}

Standard deviations in parenthesis 
Table 2

Marginal Effects from Bootstrapped OLS Regression Model

\begin{tabular}{|lcc|}
\hline & $\begin{array}{c}\text { Marginal Effects }{ }^{+} \mathbf{n = 4 6} \\
\text { Adjusted } \mathbf{R}^{\mathbf{2}=\mathbf{0 . 7 4 2 6}}\end{array}$ & $\begin{array}{c}\text { Bias-Corrected 95\% Confidence } \\
\text { Interval }\end{array}$ \\
\hline Number of Open Cancer Control Trials & $5.49^{*}(1.11)$ & 3.51 to 7.69 \\
Number of Newly Diagnosed Patients & $0.004(0.002)$ & -.002 to .008 \\
$\begin{array}{l}\text { Total Number of Staff } \\
\text { Staff Dedicated to Enrolling Patients in Control and Prevention Trials } \\
\text { One Staff Member Dedicated to Prevention and Control Trials }\end{array}$ & $.848(.74)$ & -.360 to 2.64 \\
$\quad$ More Than One Staff Member Dedicated to Prevention and Control Trials & $-22.16(20.64)$ & -70.87 to 14.09 \\
$\begin{array}{l}\text { Recognition of Physicians for Enrolling Patients in Clinical Trials } \\
\text { Provides Recognition }\end{array}$ & $29.98^{*}(15.22)$ & .816 to 60.31 \\
Expectations for Physicians to Enroll Patients in Clinical Trials & $25.35^{*}(11.65)$ & 3.43 to 47.72 \\
$\quad$ Has Expectation & & -34.23 to 34.39 \\
Constant & $3.47(17.76)$ & -71.65 \\
\hline
\end{tabular}

${ }^{+}$Bootstrapped standard errors in parentheses

Indicates significant at the .05 level 